\section{SAT0028 THE IMPORTANCE OF PROPER HANDLING OF HUMAN SYNOVIAL FLUID FOR ARTHRITIS RESEARCH}

H. Brouwers ${ }^{1}$, J.H. von Hegedus ${ }^{1}$, R.E.M. Toes ${ }^{1}$, T.W.J. Huizinga ${ }^{1}$, M.A. Giera ${ }^{2}$, M. Kloppenburg ${ }^{1}$, A. loan-Facsinay ${ }^{1}{ }^{1}$ Rheumatology; ${ }^{2}$ Center for Proteomics and Metabolomics, Leiden University medical Center, Leiden, Netherlands

Background: Synovial fluid (SF) is commonly used for diagnostic and research purposes since it is easily obtained and is thought to represent the local inflammatory environment. Common practice to obtain insight in the disease pathogenesis of arthritic diseases is the characterisation and quantification of synovial fluid cells and the measurement of inflammatory mediators. SF has a complex composition, containing hyaluronic acid which makes the fluid viscous and non-homogeneous, hampering subsequent analyses. Hyaluronic acid can be broken down by the addition of hyaluronidase, however, this is not commonly used in arthritic research.

Objectives: To determine the effect of hyaluronidase treatment on quantification and identification of SF cells and soluble mediators.

Methods: SF was obtained from twelve arthritis patients after knee aspirations as part of standard clinical care. Nine patients were diagnosed with rheumatoid arthritis, two with osteoarthritis and one with juvenile idiopathic arthritis. For cell analysis, synovial fluid was first centrifuged and the pellet was separated from the fluid. The fluid was subsequently treated with hyaluronidase and centrifuged again to isolate remaining cells. Cell numbers and phenotype were determined using flow cytometry. For soluble mediator measurements, $6-10$ aliquots were taken and treated as represented in figure 1 resulting in set 1 and set 2 . Set 1 contains replicates taken form SF before hyaluronidase treatment while set 2 mimics replicates which are taken after hyaluronidase treatment. Interleukin (IL) -8 was measured by ELISA and a total of seven fatty acid and oxidised fatty acid levels were determined using LC-MS/MS in all aliquots.

Results: Between $0.8 \%-70 \%$ of immune cells (median $5 \%$ ) are lost when the SF is not treated with hyaluronidase. This percentage is higher for $\mathrm{T}$ and $\mathrm{B}$ cells: $7 \%-$ $85 \%$ (median $22 \%$ ) and $7 \%-88 \%$ (median $23 \%$ ), respectively. To assess the variation between the soluble mediator concentrations in set 1 and set 2, the coefficients of variation (CV) of the replicate measurements were compared. Aliquots in set 2 showed a lower $\mathrm{CV}$ for the oxidised lipids 17-HDHA, leukotriene $\mathrm{B}_{4}$ and prostaglandin $\mathrm{E}_{2}$ for all patients tested. For IL-8, the oxidised lipid 15-HETE, and fatty acids arachidonic acid (AA), eicosapentaenoic acid (EPA) and docosahexaenoic acid (DHA), it did not matter whether the aliquots were taken before or after hyaluronidase treatment. While in general the average levels of the soluble mediators tested were comparable between set 1 and set 2, we did observe large differences in IL-8 and AA levels in two independent patients.

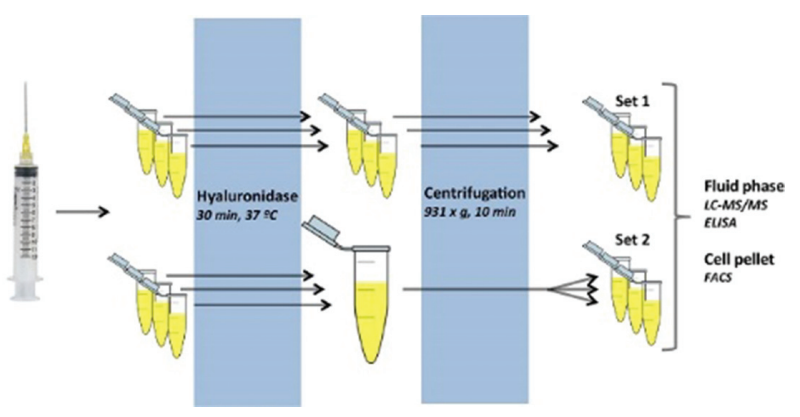

Conclusions: Up to $70 \%$ of the synovial fluid cells are missed in analysis when the $\mathrm{SF}$ is not treated with hyaluronidase before cell isolation, leading to erroneous conclusion especially when investigating rare cells populations like antigen specific B or T cells, when searching for novel cell populations, or when correlations are made between clinical parameters and cell numbers. In addition, some cytokine as well as lipid levels determined in SF without hyaluronidase treatment might not accurately reflect their actual concentrations in SF.

Disclosure of Interest: None declared

DOI: 10.1136/annrheumdis-2018-eular.6587

\section{SAT0029 IMPACT OF GLUCOCORTICOIDS ON SIRTUIN 1 EXPRESSION AND PROINFLAMMATORY CYTOKINE PRODUCTION IN RATS WITH ADJUVANT-INDUCED ARTHRITIS}

D. Wendling ${ }^{1}$, W. Abbas ${ }^{2}$, A. Kumar ${ }^{2}$, S. Pasquereau ${ }^{2}$, F. Verhoeven ${ }^{1}$, C. Prati ${ }^{1}$, C. Demougeot ${ }^{3}$, G. Herbein ${ }^{2} .{ }^{1}$ Rheumatology, CHRU Besançon; ${ }^{2} E A 4266$ EPILAB; ${ }^{3}$ EA4267 Pepite, University BFC, Besançon, France

Background: Sirtuin 1 (SIRT1) is a class III histone deacetylase which could play a critical role in several biological functions including lifespan, stress, and inflammation.

Objectives: Our main objective was to evaluate SIRT1 expression in periphera blood mononuclear cells (PBMCs) isolated from rats with adjuvant induced arthritis (AIA) treated or not with low and high doses of glucocorticoids (GCs). Our second aim was to determine the production of proinflammatory cytokines such as tumour necrosis factor alpha (TNF), interleukin-1 beta (IL-1) and interleukin-6 (IL6) in tissues (liver and spleen) of these rats, and to assess a potential correlation between SIRT1 expression and the production of cytokines in tissues of rats with AIA treated or not with low and high doses of glucocorticoids.

Methods: We performed a study on rats with AIA treated with low and high doses of prednisolone or left untreated. The SIRT1 expression was quantified in PBMCs using western blotting. Levels of proinflammatory cytokines TNF, IL-1 and IL-6 were assessed in liver and spleen from rats with AIA treated with GCs or left untreated using an ELISA assay.

Results: SIRT1 expression was increased in PBMCs isolated from rats with AIA treated with LD-GCs and HD-GCs compared to untreated animals. In culture, resveratrol enhanced the SIRT1 expression in PBMCs isolated from rats with AIA in a dose-dependent manner. We observed increased levels of TNF alpha in liver (correlated with SIRT1 expression) and decreased IL-6 levels in spleens (negatively correlated with SIRT1 expression) from rats with AIA treated with LD-GCs and HD-GCs compared to controls.

Conclusions: SIRT1 expression is increased in PBMCs isolated from rats with AIA and treated with GCs parallel to decreased levels of IL-6 in spleen and increased levels of TNF in liver. This study shows that the SIRT 1 expression is measurable in circulating PBMCs of rats with AIA and is increased under GC treatment. The potential epigenetic effect of GCs on production of proinflammatory cytokines in tissues, namely liver and spleen, is also studied.

Disclosure of Interest: None declared

DOI: 10.1136/annrheumdis-2018-eular.2752

\section{SAT0030 \\ VISFATIN DOWN-REGULATES GROWTH PROMOTING LNCRNA H19 IN OSTEOGENIC DIFFERENTIATION OF MESENCHYMAL STROMAL CELLS}

D.M. Küppers ${ }^{1}$, L. Tsiklauri ${ }^{1}$, M.-L. Hülser ${ }^{1}$, K. Frommer ${ }^{1}$, S. Rehart ${ }^{2}$, U. MüllerLadner', E. Neumann ${ }^{1} .{ }^{1}$ Dept. of Rheumatology and Clinical Immunology, JustusLiebig-University Gießen, Campus Kerckhoff, Giessen; ${ }^{2}$ Dept. of Orthopaedics and Trauma Surgery, Agaplesion Markus Hospital, Frankfurt/Main, Germany

Background: Destructive bone diseases like osteoarthritis and osteoporosis are causes of chronic pain and disability. Mesenchymal stromal cells (MSC) are key regulators for bone homeostasis due to their potential for osteogenic differentiation, preventing bone loss and promoting tissue repair. Pro-inflammatory factors including adipokines such as visfatin alter the osteogenic potential of MSCs and may contribute to the shift towards adipogenic differentiation observed in osteoporosis. Long non-coding RNA H19 is one of the first IncRNAs discovered and best understood e.g. in embryonic growth and tumour formation. IncRNAs interact directly with DNA, RNA as well as proteins and fill a regulatory gap between the ribonucleic and protein world. $\mathrm{H} 19$ upregulation was shown in osteogenic differentiation of $\mathrm{MSCs}^{1}$. $\mathrm{H} 19$ increases the osteogenic potential by acting as a sponge for miRNA 675, 141 and 22, influencing TGF $\beta 1$ and Wnt/ $/$-catenin pathways ${ }^{2,3}$ $\mathrm{H} 19$ may hence be involved in MSC mediated bone homeostasis.

Objectives: To analyse the link between adipokines and bone remodelling in destructive bone disease. To investigate the influence of adipokines on MSCs and on the expression of IncRNA H19 during osteogenesis.

Methods: Commercial human MSCs (hMSC) and primary human MSCs (phMSC) from osteoarthritis patients after knee replacement surgery were treated with differentiation medium to induce osteogenic differentiation (OD). Matrix min eralization was quantified after 21 days of OD by Alizarin Red. Expression of $\mathrm{H} 19$ 